Scalable Computing: Practice and Experience, ISSN 1895-1767, http://www.scpe.org

(C) 2021 SCPE. Volume 22, Issues 2, pp. 89-91, DOI 10.12694:/scpe.v22i2.1939

\title{
INTRODUCTION TO THE SPECIAL ISSUE ON ARTIFICIAL INTELLIGENCE FOR SMART CITIES AND INDUSTRIES
}

\author{
ASHUTOSH SHARMA* PRADEEP KUMAR SINGH; WEI-CHIANG HONG‡ GAURAV DHIMAN§ AND ADAM SLOWIK $₫$
}

Smart Cities and Artificial Intelligence offers an intensive evaluation of how the smart city establishments are made at different scales through automated thinking headways, for instance, geospatial information, data examination, data portrayal, clever related things, and quick natural frameworks handiness. Progressing propels in electronic thinking attract us closer to making a persistent reproduced model of human-made and trademark structures, from urban regions to transportation establishments to utility frameworks. This continuous living model empowers us to all the bound to manage and improve these working structures, making them dynamically watchful. Keen Cities and Artificial Intelligence gives a multidisciplinary, joined procedure, using speculative and applied bits of information, for the evaluation of savvy city situations. This special issue shows how the mechanized and physical universes are associated inside this organic framework, and how nonstop data arrangement is changing the possibility of our urban as well as industrial condition. It gives a fresh sweeping perspective on the natural framework designing, advances, and parts that include the masterminding and execution of sharp city and industry establishments. This special issue also shows how the computerized and physical universes are connected inside this biological system, and how continuous information assortment is changing the idea of our urban and industry condition. It gives a crisp all-encompassing viewpoint on the biological system engineering, advances, and parts that involve the arranging and execution of keen city and industry foundations. After following double blind peer review for all the submitted manuscripts across the globe, and after the rigorous review process, revision and based on final recommendations of the reviewers and editorial team, finally 17 manuscripts have been accepted for publication. A brief about each accepted manuscript for this Special Issue is as underneath.

The first manuscript is entitled on "Research on Construction Cost Estimation Based on Artificial Intelligence Technology" by Ghafoor et al.. This paper determines the application of grey system theory, to optimize the estimation model using Back Propagation (BP) neural network. The viability of the method established in this paper, is tested by collecting the engineering cost data in Zhengzhou city and comparing between the standard BP neural network and the Gray BP neural network methods. The results show that the average error of the Gray system theory optimized BP neural network model designed in this paper is $2.33 \%$. The Gray BP neural network model helpful for quickly estimate the project cost, with high accuracy rate.

The second manuscript is entitled on "An IoT and Blockchain Approach for the Smart Water Management System in Agriculture" by Ghafoor et al.. In this article, IoT based smart water management system is designed for the agriculture which ensures the effectiveness of the agriculture water management. A system is implemented for the agriculture water management through the real time data collection. The obtained result shows the data, that updates the water monitoring interface with the varying number of hours.

The third manuscript is entitled on "Design and Research on the Intelligent System of Urban Rail Transit Project Based on BIM+GIS" by Pljonkin et al.. The urban rail transit projects are linear projects, they not only span long lines, multiple regions, involve multiple disciplines, and are difficult to coordinate, but also have complex surrounding environments and high safety requirements. Therefore, their needs for integrated

\footnotetext{
*Institute of Computer Technology and Information Security, Southern Federal University, Russia

${ }^{\dagger}$ Department of Computer Science, KIET Group of Institutions, Delhi-NCR, Ghaziabad, Uttar Pradesh, India

$\ddagger$ School of Education Intelligent Technology, Jiangsu Normal University, China

$\S$ Department of Computer Science, Government Bikram College of Commerce, India

『Koszalin University of Technology, Faculty of Electronics and Computer Science Department of Computer Engineering, Poland
} 
construction and operation applications are more concentrated. To solve the problems of data isolation, single display form, abnormal situation notification and delayed processing in urban rail transit construction monitoring, combined with GIS+BIM technology, a complete set of construction monitoring information management process and data organization plan is proposed, and the development is oriented.

Tomar et al. contributes fourth article entitled "An IoT and Blockchain Approach for Food Traceability System in Agriculture". In this paper, food quality problems are discussed, and the food traceability system is proposed which is based on the Internet of Things (IoT) and blockchain technique for agricultural products. The consortium blockchain is utilizing as the basic network and the traceability system can achieve more reliable and trustable devices.

Zhou et al. contributes fifth article entitled "Design and Application of College Online Education Platform Based on WEBRTC". In this paper, a media server cluster load balancing algorithm based on consistency hash algorithm and genetic algorithm is proposed. This paper is focused on designing and practical implementation of a fusion communication platform combined with WebRTC and related technologies to deliver online education system in colleges and universities. The media server cluster load balancing strategy proposed in this paper can ensure the cluster overall load balancing. At the same time, the node weight can be dynamically adjusted according to the real-time state of the clusters. The outcomes obtained justifies the efficiency and practicability of the proposed methodology.

Ikbal et al. contributes sixth article entitled "Research on Data Security Detection Algorithm in IoT Based on K-Means". In this paper, an efficient data intrusion detection algorithm based on K-means clustering is proposed. Also, this paper proposes a network node control method based on traffic constraints to improve the security level of the network. Simulation experiments show that compared with traditional password-based intrusion detection methods; the proposed method has a higher detection level and is suitable for data security protection on the Internet of Things.

Lin et al. contributes seventh article entitled "Network Virus and Computer Network Security Detection Technology Optimization". In this paper, the structural model of network security detection and monitoring system is established in a proactive way. The function of each component is described, and the design model is introduced to conduct comprehensive and effective automatic security detection on the client. Each layer of the network is used to find and avoid the system from being attacked. The observed example shows that the flow rate of information in and out of the network is relatively stable, with few changes, and the rate of change is close to zero per unit time.

Yang et al. contributes eighth article entitled "A Detailed Study on GPS and GIS Enabled Agricultural Equipment Field Position Monitoring System for Smart Farming". In this paper, the nonlinear programming method has been adopted to study the agricultural machinery operation, optimal allocation, and scale management. This paper investigates the crop planting area, mechanization level, production situation, and technological process of corn planting mode. The results show that in the corn production, 11.195 million yuan of maize production value has been optimized.

Mi et al. contributes ninth article entitled "Design of Intelligent Building Scheduling System for Internet of Things and Cloud Computing". In this paper, an improved ant colony algorithm is presented to remove the shortcomings of the existing ant colony algorithm with slow speed and fall into local optimum. The improved ant colony algorithm is transplanted into cloud computing environment. The advantages of fast computing and high-speed storage of cloud computing can realize the real-time resource scheduling of building equipment. The experimental results present that the improved ant colony algorithm can obviously improve the efficiency of resource scheduling in cloud computing environment.

Sharma et al. contributes tenth article entitled "Research on TCP Performance Model and Transport Agent Architecture in Broadband Wireless Network". This paper proposes an improved scheme of TCP proxy acknowledgement based on Automatic Repeat Request (ARQ), which improves throughput, reduces delay, and saves uplink bandwidth of wireless link. The substantial improvement is observed during the experimentation as processing efficiency of protocol. The observed results revealed that overall processing time for each packet is approximately equals to one fourth of the transfer control protocol and the reduction of $59 \%$ is also observed in the utility of resources.

Paul et al. contributes eleventh article entitled "Research on Multi-Agent Systems in a Smart Small Grid for 
Resource Apportionment and Planning". The energy saving system is presented in this paper which also adapts to the inhabitant's preferences apart from environmental conditions consideration. The energy consumption of $40 \%$ is obtained and in the inhabitants' behaviour pattern, the algorithm was specialized. The $16.89 \%$ of reduction is obtained by the existing system and it was focused to obtain the agreement between the system and users for user preference satisfaction and the energy optimization is also performed at the same time.

Srivastava et al. contributes twelfth article entitled "Study and Research on IoT and Big Data Analysis for Smart City Development". In this paper, a complete system of various types of IoT-based smart systems like smart home, vehicular networking, and smart parking etc., is proposed for data generation. The Hadoop ecosystem is utilized for the implementation of the proposed system. The evaluation of the system is done in terms of throughput and processing time. The proposed technique is $20 \%$ to $65 \%$ better than the existing techniques in terms of time required for processing. In terms of obtained throughput, the proposed technique outperforms the existing technique by $20 \%$ to $60 \%$.

Mohan et al. contributes thirteenth article entitled "Cloud Based Resource Scheduling Methodology for Data-Intensive Smart Cities and Industrial Applications". This article presents an effective and time prioritization based smart resource management platform employing the Cuckoo Search based Optimized Resource Allocation (CSO-RA) methodology. The proposed (CSO-RA) system is compared with the current methodologies like particle swarm optimization (PSO), ant colony optimization (ACO) and genetic algorithm (GA) based optimization methodologies and the viability of the proposed framework is established. The percentage of optimality observed for CSO-RA algorithm is $97 \%$ and overall resource deployment rate of $28 \%$ is achieved using CSO-RA method which is comparatively much better than PSO, GA and ACO conventional algorithms.

Balyan et al. contributes fourteenth article entitled "Research on Mobile User Interface for Robot Arm Remote Control in Industrial Application". This article proposed system includes various modules like a robot arm, a controller module, and a remote mobile operating application for visualizing the robot arm angles having real time applicability. Augmented reality (AR) is utilized for robot control WIFI communication and the robot angle information is obtained for varying real time environment. The simulation results are obtained for various assessment indicators and effectual outcomes are achieved with $98.03 \%$ accuracy value and 0 . 185 , 0.180 of error and loss values for training phase. The accuracy value of $97.65 \%$ is achieved for testing phase with corresponding 0.209 and 0.190 minimum error and loss values. The proposed platform provides the feasible and reliable outcomes in the real time environment for real time manufacturing industry applications.

Kumar et al. contributes fifteenth article entitled "A Cluster Based Intelligent Method to Manage Load of Controllers in SDN-IoT Networks for Smart Cities". This paper proposes Grey Wolf Optimization Affinity Propagation (GWOAP) Algorithm to balance the traffic load of controller an intelligent cluster based when deploying the multiple controllers in SDN-IoT enabled smart city networks. The proposed algorithm is simulated and the experimental results able to calculates the minimum overall communication cost in comparison with Genetic Algorithm (GA), Particle Swarm Optimization (PSO) and Affinity Propagation (AP). The proposed GWOAP better balance the IoT enabled smart switches among clusters and node equalization is balanced for each controller in deployed topology. By using the proposed methodology, the traffic load of IoT enabled devices in smart city networks intelligently better balance among controllers.

Ziyad et al. contributes sixteenth article entitled "Novel emergency rapid response to epileptic Seizures for patients in self-driving cars". In the proposed framework, on receiving a seizure alert from wearable device of the patient, the car is stopped, and an alert is sent to the patient's family as well as registered hospital. The proposed framework is an attempt to the future applications, especially in context to the smart healthcare facilities. We hope that the quality research work published in this special issue will be helpful to upcoming research works and to the community at large.

Agarwal et al. contributes seventeenth article entitled "Enhanced secure ATM authentication using NFC Technology and IRIS verification". This manuscript covers a solution to the existing PIN based authentication problems in ATM cards. In contrast to standard architecture, the proposed solution incorporates NFC enabled smartphones as a substitute for physical card and Iris based authentication for PIN. 